Max Bense

\title{
Ausgewählte Schriften
}

Band 2

Philosophie der Mathematik, Naturwissenschaft und Technik 


\section{Max Bense}

\section{Ausgewählte Schriften in vier Bänden}

Herausgegeben und mit Einleitung, Anmerkungen und Register versehen von Elisabeth Walther 


\title{
Max Bense
}

\section{Ausgewählte Schriften}

\author{
Band 2 \\ Philosophie der Mathematik, \\ Naturwissenschaft und Technik
}


Dieses Werk wurde unterstïtzt durch:

Baden-Württembergische Bank AG

Stiftung Landesgirokasse

Landeshauptstadt Stuttgart

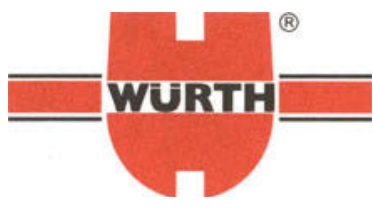

\section{Dieses Werk wurde gefördert mit Mitteln der Adolf Würth GmbH \& Co. KG.}

Die Deutsche Bibliothek - CIP-Einheitsaufnahme

Bense, Max:

Ausgewählte Schriften : in vier Bänden / Max Bense. Hrsg. und mit Einl., Anm. und Reg. vers. von Elisabeth Walther. - Stuttgart ;

Weimar : Metzler

ISBN 978-3-476-01564-8

Bd. 2. Philosophie der Mathematik, Naturwissenschaft und

Technik. - 1998

ISBN 978-3-476-01566-2

ISBN 978-3-476-01566-2

ISBN 978-3-476-03715-2 (eBook)

DOI 10.1007/978-3-476-03715-2

Dieses Werk einschließlich aller seiner Teile ist urheberrechtlich geschützt. Jede Verwertung außerhalb der engen Grenzen des Urheberrechtsgesetzes ist ohne Zustimmung des Verlages unzulässig und strafbar. Das gilt insbesondere für Vervielfältigungen, Übersetzungen, Mikroverfilmungen und die Einspeicherung und Verarbeitung in elektronischen Systemen.

(C) 1998 Springer-Verlag GmbH Deutschland

Ursprünglich erschienen bei J. B. Metzlersche Verlagsbuchhandlung und Carl Ernst Poeschel Verlag GmbH in Stuttgart 1998 


\section{Inhalt}

Elisabeth Emter: Einleitung $\ldots \ldots \ldots \ldots$ VII

Quantenmechanik und Daseinsrelativität (1938) . . . . 1

Konturen einer Geistesgeschichte der Mathematik.

Die Mathematik und die Wissenschaften (1946) . . . . . . 103

Konturen einer Geistesgeschichte der Mathematik II.

Die Mathematik in der Kunst (1949) . . . . . . . . . 233

Kybernetik oder Die Metaphysik einer Maschine (1951) . 429

Anmerkungen . . . . . . . . . . . . . . . . . . 449

Namenregister . . . . . . . . . . . 475 


\title{
Einleitung
}

\author{
Von Hause aus Physiker \\ und Mathematiker. \\ Der Philosoph Max Bense
}

1989 in einem Interview auf seine Philosophie hin angesprochen, antwortete Max Bense: "Ich bin von Hause aus Physiker und Mathematiker, dann wissen Sie ja, was los ist « ${ }^{1}$ Sein Gesprächspartner August Häfner fand die Antwort "trocken ", und das nicht ganz zu unrecht, denn wissen wir wirklich, was los ist, wenn ein Philosoph den Ordnungsraum, in dem sich sein Wissen und Denken konstituiert hat, mit den Koordinaten Mathematik und Physik bestimmt? Für Häfner erklärte sich aus Benses Präferenz für die exakten Wissenschaften die vehemente Verteidigung der Rationalität und die strikte Ablehnung von allem, was die Ratio mißachtet. Doch damit sind noch keineswegs alle Modalitäten und Konsequenzen erschlossen, die das Denken eines Grenzgängers zwischen den mathematisch-naturwissenschaftlichen und den philosophisch-literarischen Diskursen auszeichnen.

Als Bense Philosophie, Mathematik und Physik zu studieren anfing, hatten sich die Grundlagen der naturwissenschaftlichen Erkenntnis auf revolutionierende Weise geändert. ${ }^{2}$ Mit der speziellen und allgemeinen Relativitätstheorie begannen sich neue Vorstellungen von Raum und Zeit sowie eine neue Auffassung vom Universum durchzusetzen. Indem die Relativitätstheorie verlangt, daß der Standpunkt des Beobachters einbezogen werden muß, erschütterte sie die Fundamente der klassischen Physik zwar erheblich, setzte sie jedoch nicht völlig außer Kraft. Die Entdeckung einer bis dahin noch unbekannten Naturkonstanten, des Wirkungsquantums, durch Max Planck im Jahre 1900 leitete dagegen einen spektakulären Wandel in den Denkkategorien der Physik ein.

Die Beobachtungen im mikrophysikalischen Bereich offenbarten zum einen, daß mit einer grundsätzlichen Stetigkeit des $\mathrm{Na}$ turgeschehens nicht gerechnet werden kann. Zum anderen stellte man fest, daß Strahlung und Materie eine Doppelnatur auf- 
weisen. Beide lassen sich sowohl als Teilchen als auch als Wellen beschreiben. Diese der Alltagserfahrung sowie der klassischen Logik durch die Aufhebung des Identitätsprinzips widersprechenden Erkenntnisse erfuhren mit dem Ausbau der Quantenmechanik noch eine Radikalisierung. Als Werner Heisenberg 1926 die Unschärferelation formulierte, mußten die Physiker zu ihrem Entsetzen feststellen, daß bis zu diesem Zeitpunkt unbezweifelte Grundfesten ihrer Wissenschaft in Frage gestellt wurden. Die Unbestimmtsheitsrelation legt in Form eines Naturgesetzes fest, daß im mikrophysikalischen Bereich keine raumzeitliche Beschreibung eines Bewegungsvorganges im klassischen Sinne möglich ist. Seit Galilei und Newton waren die Physiker davon ausgegangen, daß der Zustand eines Systems exakt vorhergesagt werden kann, wenn man über alle Details des Zustandes zu einem früheren Zeitpunkt verfügt. Entgegen dieser Prämisse der klassischen Physik muß im Bereich der kleinsten Teilchen anhand von Wahrscheinlichkeitsaussagen mit Hilfe der Statistik operiert werden, weil nie alle Details des Zustandes eines mikrophysikalischen Systems durch Messungen in beliebiger Genauigkeit ermittelt werden können. Daß man gezwungen war, die Statistik zu Hilfe zu nehmen, hätte die Physiker nicht allzusehr beunruhigt, denn auf sie griff man auch bei der Betrachtung von Gasen zurück. Weil es meßtechnisch nicht möglich ist, jedem einzelnen Gasmolekül eine bestimmte Geschwindigkeit zuzuschreiben, ermittelt der Physiker statistische Mittelwerte und erhält auf diesem Weg zutreffende Werte zur Bestimmung des Gasdruckes. Doch in Bezug auf die Welt der kleinsten Teilchen lag kein meßtechnisches Unvermögen vor. Die Quantenphänomene selbst zwingen zur Statistik.

Die Physiker waren auf eine Erkenntnisschranke gestoßen, die ihnen der atomare Bereich auferlegte. Die Welt der kleinsten Bestandteile war zwar der mathematischen Beschreibung zugänglich, aber sie konnte unmöglich im Sinne der gewohnten Realitätsvorstellungen beschrieben werden. Um dies mit dem bis dahin gültigen Bild der Natur in Einklang zu bringen, mußten die Physiker die Grenzen ihrer Disziplin überschreiten und erkenntnistheoretische sowie ontologische Fragestellungen reflektieren. Die Beobachtungen schienen nur dann innerhalb eines theoretischen Gebäudes schlüssig erklärt werden zu können, wenn 
das traditionelle Verständnis der Subjekt-Objekt-Relation innerhalb des Beobachtungsprozesses, alte Konzepte des Substanzbegriffes und der Glauben an einen lückenlosen Ursache-WirkungsZusammenhang neu überdacht wurden. Es entspann sich im Kreise der Physiker in den Jahren nach der Formulierung der Unschärferelation eine Diskussion, inwieweit eine vom Beobachter unabhängige, d.h. objektive Beobachtung überhaupt möglich ist und inwieweit die Welt der kleinsten Teile, aus der man sich unsere Erfahrungswelt aufgebaut gedacht hatte, von Akausalität bestimmt ist, ja inwieweit überhaupt noch an der Vorstellung einer gegenständlichen Materie festgehalten werden kann.

Die Physiker diskutierten jedoch nicht nur in ihren eigenen Reihen. In einer Fülle von allgemeinverständlichen Beiträgen in Zeitungen und Zeitschriften versuchten sie auch den nicht-naturwissenschaftlich ausgebildeten Zeitgenossen die neuen Resultate der Physik und die möglichen Erschütterungen, die sie dem naturwissenschaftlichen Weltbild beifügten, nahezubringen. Die zeitgenössischen Philosophen wie Ernst Cassirer, Moritz Schlick oder Ortega y Gasset wurden schnell aufmerksam, als sie bemerkten, daß die Physiker ihr angestammtes Terrain verließen und die altbekannten philosophischen Probleme auf einer neuen Stufe naturwissenschaftlicher Erkenntnisse zu erörtern begannen. ${ }^{3}$ Auch der Student Max Bense war von den Entwicklungen, die sich in der Physik vollzogen, fasziniert. Er verfolgte die Resultate der physikalischen Forschung nicht nur, sondern er berichtete immer wieder über sie, so vor allem auf den Wissenschaftsseiten der überregionalen Kölnischen Zeitung. ${ }^{4}$

1937 veröffentlichte Bense in der Kölnischen Zeitung einen Beitrag mit dem Titel "Was ist Substanz? " 5 Bevor er die neuesten Erklärungsansätze der Physiker darlegt, leitet er seinen Artikel mit einer allgemeinen Betrachtung ein. Er verweist darauf, daß die Frage nach der Substanz eine "alte Lieblingsfrage « der Philosophen sei, die dennoch nie eine befriedigende Antwort gefunden habe. Die Ursache hierfür sieht er in der feindlichen Spannung, die zwischen Philosophen und Physikern herrsche. Letztere klebten allzu eifrig an nackten Tatsachen. Die anderen glitten aus ihrer Liebe zur Spekulation ins Reich der Phantasie ab. Seit dem Entstehen der Quanten- und der Relativitätstheorie glaubt er 
jedoch erste Schritte in Richtung einer "notwendigen Zusammenarbeit zwischen dem exakten Naturwissenschaftler und dem Philosophen « erkennen zu können. Die Tatsache, daß die Resultate der modernen Physik direkt in erkenntnistheoretische und ontologische Probleme führten, schien ihm die beste Voraussetzung für solch einen Brückenschlag.

Bense trat bereits in seiner Studienzeit für eine Verbindung von Philosophie und mathematisch-exakter Naturerkenntnis ein. Doch er begnügte sich keineswegs mit dem Plädoyer für eine Zusammenarbeit. Mit einer im Rahmen seiner Promotion verfaßten Abhandlung machte er sich daran, selbst an der von ihm geforderten Zusammenführung von Philosophie und Naturwissenschaft mitzuwirken. In seiner 1938 veröffentlichten Dissertation Quantenmechanik und Daseinsrelativität versucht er eine philosophische Grundlage der modernen Physik zu erarbeiten, indem er die Quantenphänomene vor dem Hintergrund von Max Schelers phänomenologischem Konzept eines "Stufenreichs der Daseinsrelativität der Gegenstände ${ }^{6}{ }^{\text {deutet. }}$

Scheler entwickelte vor allem in seinem Spätwerk und seinen erst posthum veröffentlichten Manuskripten die Vorstellung einer relativen Erkenntnis, die nicht auf der Subjektivität des erkennenden Subjekts basiert. Gegenüber Kants Annahme, daß sich Wirklichkeit nur in Form der sinnlichen Erfahrung manifestiert, während das "Ding an sich " dahinter unsichtbar bleibt, postuliert Scheler eine Realität, in der eine erkennbare, aber gleichzeitig vom Denken unabhängige Ordnung herrscht. Die im Erkenntnisprozeß aufgedeckten Wesensstrukturen korrespondieren mit den in der Realität enthaltenen, außerhalb des Bewußtseins liegenden Strukturen. ${ }^{7}$ Wissen ist für ihn weder eine Transformation noch eine Abbildung der Wirklichkeit im Bewußtsein, sondern Produkt einer ständigen Wechselwirkung zwischen Bewußtsein und Realität. Es ist "Teilnahme und Teilhabe eines konkret Seienden (des wissenden Subjekts) an dem Sosein von anderem konkret Seiendem «. Daseiend bedeutet für ihn zwar außerhalb und unabhängig des Bewußtseins daseiend, aber »gleichwohl nur relativ, d.h. abhängig vom Dasein eines so oder anders seienden und (im objektiven Sinne) bestimmten realen Trägers dieses Wissens ${ }^{8}$. Je nachdem, »auf was an dem teilhabenden Seienden, zum Beispiel dem Menschen, die betreffende Stufe der Daseins- 
relativität eben sdaseinrelativ ' ist und auf was nicht mehr; zum Beispiel auf den Menschen als Körperding überhaupt, als Lebewesen überhaupt, als endlichen erkennenden Geist überhaupt, oder relativ im Universum der ausgedehnten Körper oder auf keinen besonderen Standort mehr " ${ }^{9}$, enthüllt sich dem Denken eine ganz spezifische Wirklichkeitsstruktur. Die Sonne, die ein wahrnehmendes Subjekt von einem Fenster aus betrachtet, kann auf den augenblicklichen Bildinhalt begrenzt als roter Feuerball am Himmel angesehen werden. Sie kann als Sonnenball, der hinter den Bergen verschwindet, ohne dabei seine Identität aufzugeben, verstanden werden. Sie kann aber auch unabhängig vom Standpunkt des Betrachters auf der Erde und seinen psychophysischen Eigenschaften als Sonne im Sinne der astronomischen Erkenntnisse, als Körper in Wechselbeziehung zu anderen Körpern im Universum, bis hin zum chemischen Aufbau oder den nur noch mathematisch beschreibbaren mikrophysikalischen Strukturen begriffen werden.

Anhand Schelers Lehre von der Daseinsrelativität entwirft Bense ein Erklärungsmodell der Doppelnatur von Strahlung und Materie. Nach den Anschauungen der klassischen Physik, die an der Erfahrung der Alltagswelt geschult war, stellte es ein Paradox dar, daß Materie sowohl Eigenschaften von Wellen und von Teilchen aufwies, daß ein und derselbe Sachverhalt sich sowohl mit einem Teilchen- als auch mit einem Wellenbild beschreiben ließ. Für Bense hebt sich dieser Widerspruch zwischen den beiden Beschreibungsmodi Welle und Teilchen auf, weil er auf Schelers Theorie aufbauend davon ausgeht, daß von zwei sich widersprechenden Sätzen nur dann einer falsch sein muß, wenn sich die Sätze auf dieselbe Stufe der Daseinsrelativität beziehen. Seiner Ansicht nach sprechen die Experimente, die den Wellencharakter, und jene Experimente, die den Teilchencharakter der atomaren Erscheinung ermitteln, jeweils eine andere Stufe der Daseinsrelativität eines physikalischen Gegenstandes an. Die heisenbergschen Unschärferelationen kennzeichnen laut Bense die Schnittstelle zwischen moderner Physik und Phänomenologie, weil sie zwei Gegebenheitsstufen, Welle und Teilchen, eines einheitlichen physikalischen Tatbestandes begrenzen. Demnach hat die Quantenmechanik durch eine Verfeinerung der Experimente eine neue Stufe der Daseinsrelativität der physikalischen Wirklichkeit frei- 
gelegt. Aus dieser Sicht steht die moderne Physik nicht konträr zur klassischen Physik, sondern schließt sich ihr an und ergänzt sie.

Die Tatsache, daß die Quantenmechanik nicht in der Lage ist, ein anschauliches Modell für den Aufbau der mikrophysikalischen Welt zu liefern, sondern nur eine rein mathematische und auf Wahrscheinlichkeitsaussagen beruhende Beschreibung ihres Beobachtungsgegenstandes gibt, wertet Bense nicht als Erkenntnisverlust. In Anlehnung an Heidegger ${ }^{10}$ unterscheidet Bense zwischen einer endlichen und einer unendlichen Erkenntnis, die jeweils zwei verschiedenen Stufen der Daseinsrelativität der Gegenstandsarten entsprechen. Die klassische Physik, die ihre Gegenstände anschaulich bestimme, ordnet er der endlichen Erkenntnis zu. Die moderne Physik dagegen, die ihre Gegenstände nur noch formal, in der mathematischen Symbolsprache beschreibe, gehört für ihn zur unendlichen Erkenntnis, insofern sie das "Seiende im Entstehen-lassen «, als Möglichkeit zeige. Die mathematischen Formeln sind folglich nicht dem physikalischen Tatbestand aufgepfropft, sondern geben den geistigen Blick auf das "Ding an sich" frei.

Auflösung des Widerspruchs von Welle und Teilchen und positive Bewertung der Unanschaulichkeit sind nur zwei Punkte aus Benses Einbettung der modernen Physik in Schelers Lehre der Daseinsrelativität. Sie genügen jedoch, um auf einen wichtigen Aspekt des argumentativ-rhetorischen Aufbaus von Benses Dissertation aufmerksam zu machen. Benses Arbeit ist mit dem Ziel konzipiert, die moderne Physik als eine Fortführung der klassischen Naturwissenschaft darzustellen. Es kommt ihm darauf an zu verdeutlichen, daß die Quantenmechanik weder die Physik im speziellen oder die Rationalität im allgemeinen in eine Krise geführt hat, noch völlig losgelöst und abgehoben von der Realität und dem Menschen steht. Um diesen Ansatz richtig verstehen zu können, bedarf es einiger Hinweise auf die historische Situation, in der Benses Promotionsarbeit entstand.

Als Bense zu studieren begann, befand sich die Physik nicht nur unter dem Einfluß der Relativitäts- und Quantentheorie, sondern auch unter dem Eindruck der "Deutschen Physik «. Bereits in der Weimarer Republik hatten die Physiker Philipp Lenard und Johannes Stark begonnen, eine Wissenschaftstheorie auf 
der Basis der nationalsozialistischen Rassenideologie zu entwikkeln. Sie wurden allerdings nicht sonderlich ernst genommen. Nach 1933 erhielten die beiden jedoch als alte Parteigänger einflußreiche Stellen. Lenard wurde schon 1933 Präsident der Physikalisch-Technischen Reichsanstalt und Stark 1934 Präsident der Deutschen Forschungsgemeinschaft. Ihre Wissenschaftstheorie ging davon aus, daß nur der Arier richtige Physik betreiben kann. Unter richtiger Physik verstanden sie eine Physik, die auf physikalischen Experimenten aufbaut und für die die Theorie nur eine anregende Wirkung hat. Die experimentell gewonnenen Ergebnisse sollten in anschaulicher Weise beschrieben, gedeutet und erklärt werden. Sie sollten fest auf dem Boden der klassischen Physik stehen. ${ }^{11}$ In diesem Sinne wurde die Relativitätstheorie als großer "jüdischer Weltbluff «, als theoretische Magie und als ein Formelsystem aus völlig unbewiesenen und lückenhaften Überlegungen und ungeheuerlichen Extrapolationen diffamiert. ${ }^{12}$ Die Quantentheorie wurde ebenso wie die Relativitätstheorie wegen des hohen Grads an Mathematisierung und wegen der Unanschaulichkeit als "jüdische Physik « verschrien. Heisenbergs Unschärferelation erklärte man zu einer metaphysischen Phantasie. Die Mehrheit der deutschen Physiker, selbst jene, die Gegner der Relativitäts- und Quantentheorie waren, lehnten zwar die "Deutsche Physik «ab, dennoch schufen Lenard und seine Anhänger ein unangenehmes Klima. 1936 kulminierte die ideologische Hetze in der Beschimpfung Werner Heisenbergs, Max von Laues und Arnold Sommerfelds als "weiße Juden «, weil sie die formalistischen und unanschaulichen Tendenzen der modernen Physik in ihren Forschungen weiterverfolgten. Erst 1941 wurde aus pragmatischen Gesichtspunkten von offizieller Seite die Relativitätstheorie und die Quantentheorie mit allen mathematischen Hilfsmitteln und Formalismen als fester Bestand der Physik bestätigt. ${ }^{13}$

Im November 1936 reagierte Max Bense mit einem kritischen Artikel in der Kölnischen Zeitung auf Lenards Bestrebungen einer »Deutschen Physik « ${ }^{14}$ Was Lenard in seinen Lehrbüchern ${ }^{15}$ präsentiert, ist in seinen Augen eine Physik, die auf dem Stand von 1900 stehengeblieben ist. Indem er darauf hinweist, daß an der Entwicklung der modernen Physik »echt deutsche Männer und Forscher andrer germanischer Länder « beteiligt waren und 
daß die mathematischen Grundlagen der Relativitäts- und Quantentheorie auf "zwei unzweifelbar deutsche[n] Forschergestalten: Leibniz und Gauß « zurückgehen, versucht er zu zeigen, wie absurd die Trennung in eine jüdische und deutsche Physik ist. Den Grund für Lenards Ablehnung der modernen Physik vermutet Bense ganz woanders. Provokant stellt er die Frage, ob es Pädagogik sei, "daß man neuere Tendenzen, und zwar solche, die nicht von Juden stammen, bloß weil sie einem nicht in die eignen physikalischen Vorstellungen passen, verschweigt«. Den wahren Beweggrund von Lenards Mission für eine "Deutsche Physik " sieht er daher in einem Konservatismus, der es leicht mache, "daß jeder Dilettant, bloß weil er mathematisch unbegabt ist, mit einer Handbewegung Feldtheorie, Quantentheorie und Quantenmechanik als jüdisch ablehnen kann «.

Durch seine Argumentation, mit der sich Bense um eine richtige Einschätzung der modernen Physik als eine unabhängige Wissenschaft bemüht, gerät er bedenklich in das Fahrwasser einer rassistischen Rhetorik, vor allem dann, wenn er betont, daß die moderne Physik "trotz der durch eine falsche Universitätspolitik begünstigten jü̈dischen $W$ issenschaft eine einzigartige Kontinuität deutscher und echter europäischer Forschung bis in unsere Tage erhalten geblieben " sei. ${ }^{16}$ Seine Dissertation zeugt allerdings von einem ganz anderen Geist. Hier werden ebenso Schriften von Albert Einstein und Hermann Minkowski als auch Werke von verpönten Philosophen wie Edmund Husserl, Ernst Cassirer und Moritz Schlick zitiert. Max Scheler, der im Sinne der rassistischen Terminologie "jüdischer Mischling " war, liefert sogar die Lehre, auf der Bense seine Studie aufbaut. Er wählte die von ihm verwandte Literatur somit nicht nach rassistisch-ideologischen Gesichtspunkten aus, sondern danach, inwieweit sie ihm plausibel und einer Verteidigung der rationalen Naturwissenschaft dienlich schien. Damit fügt sich seine Dissertation in ein Konzept ein, dessen Grundstein er bereits 1935 mit seiner Abhandlung Aufstand des Geistes. Eine Verteidigung der Erkenntnis legte und in seiner Streitschrift Anti-Klages oder von der Würde des Menschen 1937 vertiefte.

Mit diesen beiden Werken bezog Bense Stellung gegen alle geistfeindlichen Tendenzen, die den Geist als Widersacher des Lebens anklagen, ohne Perspektiven dafür zu entwickeln, wie das 
Leiden am Geist überwunden werden kann. Geist und Leben, Rationalität und Existenz gehören für ihn zusammen, und er ist sich sicher, daß nur der am Geist leidet, der ihn verloren hat. Die Naturwissenschaften und die Mathematik aufgrund ihrer rational-logischen Methodik als Feinde des Lebens zu kritisieren, ist für Bense ein eindeutiges Symptom einer Geistfeindschaft, die auf dem Verlust von Geist basiert. Aus seiner Sicht sind es gerade die reine Logik, die Mathematik und die theoretische Physik, die zu einer Naturerkenntnis aus der Frage nach der menschlichen Existenz leiten und die zu einer Überwindung der Kluft zwischen Subjekt und Objekt führen. Für ihn gibt es einen Weg, der aus dem "Reich der intuitiven Wahrheiten der Husserl und Bergson in die Mitte der tiefsten Gebiete moderner Quantenphysik ", der von der »tiefen Innerlichkeit Kierkegaards zu den Relationen Heisenbergs « und zu einem Geist führt, der die Betrachtung alles Menschlichen und Außermenschlichen zu verbinden vermag. ${ }^{17}$

Es ist nicht verwunderlich, daß Bense als Verfechter der rationalen Erkenntnis 1942 unter dem Pseudonym Karl Zink ein allgemeinverständliches Buch über die Physik seiner Zeit verfaßt, in dem er vor allem über die mathematischen und theoretischen Zusammenhänge berichtet. ${ }^{18}$ Gleich zu Beginn beruft er sich auf Galilei: zusammen mit dem von Kopernikus stammenden Motto "Obgleich ich weiß, daß die Einsicht der Philosophen dem Urteil der Menge entzogen ist, [...] so halte ich doch dafür, daß man Meinungen, die von der Richtigkeit ganz entfernt sind, vermeiden müsse « ein deutlicher Fingerzeig auf die Geschichte, in der sich Erkenntnisse immer wieder gegen ideologische Denkbarrieren durchsetzten mußten ${ }^{19}$. Entsprechend betont Bense, daß Galileis Lehrbuch Discorsi e Dimostrazioni Mathematiche, das als Grundlegung der neuzeitlichen Naturwissenschaft gilt, allen »Völkern geschenkt " worden sei. Alle Völker hätten am Aufbau der Physik mitgearbeitet, kein Volk sei wegzudenken. ${ }^{20}$ Doch Bense sieht in Galilei nicht allein den Vater einer internationalen Physik, sondern den Begründer einer Methode, die auf »der Sicherheit und Gründlichkeit der Aussage ${ }^{21}$ basiert und der allein die Naturwissenschaften ihren Erfolg verdanken. Für Bense war entscheidend, daß Galileis Naturbeobachtung darauf basiert, einzelne Naturvorgänge durch Experimente aus dem Ganzen des Naturgeschehens herauszulösen, mathematisch $\mathrm{zu}$ beschreiben 
und in ihren Gesetzmäßigkeiten zu verstehen. Die Naturbetrachtung reduzierte sich auf die meßbare, auf die mathematisierte Natur.

In Benses Augen steht Galilei zusammen mit Kopernikus, Newton und Kepler am Beginn einer Entwicklung, die durch konsequente Anwendung der Mathematik zum Aufschwung der modernen Wissenschaften führte. Gleichwohl sollte man nicht dem Irrtum erliegen, die Mathematik stelle für Bense lediglich ein Hilfsmittel der Wissenschaften dar. Seine Verteidigung der modernen Physik war im wesentlichen durch die Verteidigung ihres hohen Grades an Mathematisierung geprägt. Die Verteidigung der Mathematik wiederum vollzog sich unter dem Zeichen einer Verteidigung des Geistes. Mathematik ist für Bense nicht nur eine Sprache, in der die Wissenschaften ihre Beobachtungen und Erkenntnisse fixieren. Sie ist für ihn die klarste und tiefste Form menschlichen Denkens. Parallel zu seinen Arbeiten über die moderne Physik veröffentlichte Bense immer wieder Aufsätze und Rezensionen in der Kölnischen Zeitung, in denen er Mathematiker aus verschiedenen Epochen und deren Denkleistungen oder Probleme der zeitgenössischen Mathematik vorstellte. ${ }^{22}$ Die 1939 in der von ihm selbst herausgegebenen Reihe Einheit des Wissens. Natur- und geisteswissenschaftliche Handbücher erscheinende Abhandlung Geist der Mathematik ${ }^{23}$ läßt sich einerseits als $\mathrm{Zu}$ sammenfassung und Vertiefung dieser Zeitungsbeiträge, andererseits als Einleitung seiner Konturen einer Geistesgeschichte der Mathematik lesen.

Ohne sich davon abschrecken zu lassen, daß die Mathematik bislang nur dadurch "populär" war, "daß sie gefürchtet wurde ${ }^{24}$, wagt er in diesem Werk den Versuch, dem Leser das mathematische Wissen und die mathematische Methode als eine Möglichkeit des Denkens näherzubringen, das weit über rein mathematische Probleme hinausgeht. Es geht ihm darum, darzulegen, daß Mathematik eine »elementare Äußerung des menschlichen Geistes « ${ }^{25}$ ist. Sie ist für ihn weder eine untergeordnete noch eine von anderen Manifestationen des menschlichen Geistes losgelöste Erscheinung. Alle kulturellen Tätigkeiten des Menschen, sei es auf dem Gebiet der Wissenschaft, Technik und Architektur, sei es in der Musik, Kunst, Literatur, Philosophie oder Theologie, korrespondieren mit Entwicklungen im mathe- 
matischen Denken, ja der Stand der Mathematik ist für sie entscheidend. Die "Geschichte der Mathematik als Geschichte der Entfaltung des menschlichen Geistes ${ }^{26}$ begreifend, ist es für Bense ausgeschlossen, daß eine Geistes- und Kulturgeschichte geschrieben werden kann, wenn man die Entwicklung der Mathematik nicht berücksichtigt.

Während Bense in Geist der Mathematik den Schwerpunkt seiner Darstellung auf die Herausarbeitung von vier Phasen, der Grundlegung der Mathematik durch Pythagoras, Thales und Euklid, ihrer Weiterentwicklung durch Gauß, Descartes und Leibniz, ihr Sturz in eine Krisis durch Cantor, Riemann und Lobatschewski und ihre Überführung auf eine neue Stufe von Hilbert, Russell und Whitehead legt, verlagert sich in den Konturen einer Geistesgeschichte der Mathematik sein Interesse endgültig auf epistemologische Zusammenhänge. Statt Axiome und Theoreme, Probleme und ihre Lösungen, Mathematiker und ihre Werke zu behandeln, untersucht er in den beiden Bänden die Wechselwirkung zwischen mathematischem Wissen und Gesamtwissen, das geistige Klima, dem mathematische Theoreme entwuchsen, und die Wirkungen, die mathematisch-logische Grundlagen in anderen Bereichen des Wissens und der Kultur entfalteten.

Mit seinem Gang durch das Wissen von der Antike bis in die Gegenwart zeichnet Bense die zunehmende Ablösung der mathematischen Gedankenbildung von der gegebenen und anschaulichen Wirklichkeit hin zu einem immer subtileren Grad an Abstraktion nach. Die Mathematik der Antike steht seinen Ausführungen zufolge noch zu stark unter dem Einfluß mythisch-philosophischer Ideen und ihrer Kosmologie, als daß sich eine von der sinnlichen Gegebenheit unabhängige Betrachtung der geometrischen Formen hätte durchsetzen können. Erst mit Leibniz' Entwurf einer "mathesis universalis «, mit der nicht nur die Denkprozesse innerhalb der Wissenschaft, sondern auch in der Philosophie und Theologie unter die Operationsregeln der Aussagenund Schlußlogik der Mathematik gestellt werden sollten, wird die Voraussetzung zur gänzlichen Loslösung vom Primat der Anschauung geschaffen. Im Zuge der Grundlagenkrise der Mathematik durch die mengentheoretischen Paradoxien und die Entdeckung der nichteuklidischen Geometrie behauptet sich eine Mathematik, die nur noch in mathematischen Symbolen und 
Symbolreihen über etwas spricht, das eine Leerstelle ist. Die Mathematik wurde in unserem Jahrhundert bei den mathematischen Formalisten zu einer Sprache mit bedeutungsfreien Zeichen, was die mathematischen Intuitionisten jedoch vehement ablehnten.

Auf der Grundlage seines Bildes von der Entwicklung der Mathematik deckt Bense im Verlauf seiner Abhandlung die Vernetzung mit den spezifischen Denkweisen in der Philosophie, Theologie, Kunst, Literatur und anderen Wissenschaften verschiedener Epochen auf. Die Anfänge der antiken Ornamentik, die sich an den Gesetzmäßigkeiten der Natur orientiert, sind für ihn ebenso mit der mathematischen Begriffsbildung verbunden wie die Vorliebe des Barocks für Schnörkel mit der Differentialgeometrie. Calvins Lehre der Prädestination und der Laplacesche Dämon stehen für ihn genauso mit den universalistischen Tendenzen der Mathematik in Zusammenhang wie die technische Rationalisierung des Lebens und die Herausbildung des Kapitalismus. In gleicher Weise läßt sich die Kunst und Literatur des Surrealismus im Konnex mit Paradigmen der Naturwissenschaften und in Abhängigkeit von der Grundlagenkrise der Mathematik sehen. In Einklang mit Leibniz' Idee, daß Geist Form und Form Mathematik ist, gehören für Bense Geistesgeschichte und Geschichte der Mathematik zusammen.

Die Mathematik des 17. Jahrhunderts, vor allem aber Leibniz und seine "mathesis univeralis « bilden den Dreh- und Angelpunkt der Konturen einer Geistesgeschichte der Mathematik. Indem Bense alle geistigen Manifestationen des Menschen in Bezug zur Entwicklung und Herausbildung mathematischer Theorien und Methoden setzt, stellt er sich bewußt in die Tradition eines philosophischen Programmes, das menschliches Wissen in einem formalistischen Schema erfassen will. Bereits Descartes ließ nur die Mathematik als einzig verläßliche Methode gelten, mit der sicheres Wissen über die Welt und die Menschen erlangt werden kann. Leibniz radikalisierte diesen Ansatz noch, insofern er nach einer Sprache suchte, die mit anderen Mitteln als reinen Worten Wissen darstellen sollte. Er ging von der Vorstellung aus, daß das menschliche Denken, gleichgültig, ob es sich nun in Worten, Symbolen, Bildern oder Zahlen vollzieht, nichts anderes ist als ein Verbinden und Ersetzen von Zeichen. ${ }^{27}$ Infolgedessen 
kam es Leibniz darauf an, Zeichen zu finden, die dazu geeignet sind, alle unsere Begriffe so definitiv und genau auszudrücken, wie die Arithmetik Zahlen oder die geometrische Untersuchung Geraden beschreibt. Es schien ihm sicher, daß alle menschlichen Aussagen und Behauptungen wiedergegeben und alle logischen Überlegungen exakt untersucht werden könnten, wenn es gelänge, komplizierte Gedanken durch eindeutige Zeichenketten darzustellen und Gesetze für vernünftiges Schließen als Regeln für den Umgang mit den Ketten anzugeben. Nach Leibniz sollte jedem gegebenen Begriff eine Zahl zugeordnet werden. Ein Begriff, der aus einer Kombination anderer Begriffe besteht, sollte durch das Produkt der Zahlen der anderen Begriffe dargestellt werden. Das Prinzip ist einfach: Wird der Begriff »Lebewesen « durch die Zahl 2 ausgedrückt und der Begriff "vernünftig " durch die Zahl 3, dann kann der Begriff "Mensch " durch die Zahl 6 als Produkt aus 2 und 3 ausgedrückt werden. Diese Idealsprache, die "lingua characteristica universalis «, und ihr Regelsystem, das "calculus ratiocinator «, basierten darauf, daß alle logischen Herleitungen auf Algebra reduziert werden. Leibniz war davon überzeugt, daß durch einen Ausbau der von ihm konzipierten Universalsprache, in der Denken und Mathematik vereint sind, alle strittigen Fragen sowohl in der Philosophie als auch in der Theologie gelöst werden können.

Wie Bense in den Konturen einer Geistesgeschichte der Mathematik immer wieder hervorhebt, sind es nicht die Monadenlehre oder die Theodizee, die er zu den entscheidendsten Beiträgen von Leibniz für die Geistesgeschichte zählt. Bedeutender scheint ihm, daß Leibniz das logische Schlußverfahren zu einem Rechenprozeß transformierte, bei dem die Bedeutung der Zeichen irrelevant wird, und so die Grundlage zu einer generalisierten Mathematik, einer Mathematik schuf, die sich auch auf nicht-mathematische Gegenstände erstreckt. Erst mit der Fundierung der "mathesis universalis " auf der Basis eines widerspruchsfreien, eines tautologischen Zeichenspiels, gewinnt für Bense die Metaphysik Leibniz' von der besten aller möglichen Welten an Gehalt. In der Beherrschung möglicher und abstrakter Welten sieht er die Voraussetzung, um von einer Repräsentation und Nachahmung der vorgegebenen Wirklichkeit zu einer Realisierung des Möglichen fortzuschreiten. Die Idee einer Univer- 
salmathematik, die laut Bense seit Leibniz in den Arbeiten von Frege, Hilbert, Gödel und Russell stetig weiterwirkte, ist für ihn nichts weniger als eine ganz spezifische Art und Weise des Intellekts, sich zu dem Bestehenden, zur konkreten Wirklichkeit zu verhalten: Sie ist eine Ideologie.

In der Vorbemerkung des ersten Bandes der Konturen einer Geistesgeschichte der Mathematik stellt Bense die These in den Raum, daß die Mathematik so etwas wie eine "große, geheime Ideologie" sein könnte. Dies zu untersuchen, sei Anliegen seines Buches. Im Nachwort nimmt er diese These noch einmal auf. Er nennt sich so »leibnizisch « gesonnen, daß für ihn die Mathematik eine Ideologie ist. Das mag den Leser zunächst irritieren, wird doch heutzutage in der Alltagssprache unter Ideologie meist eine "Betrugstheorie" verstanden, die nur dann funktioniert, wenn man selber daran glaubt. ${ }^{28}$ Wer heute den Begriff "Ideologie " gebraucht, schreibt ihn meist anderen zu, nicht aber sich selber. ${ }^{29}$ Aus der ursprünglichen Bedeutung einer »Wissenschaft der Ideen " ist eine Kampfparole, eine Bezichtigungsformel geworden. Auf den ersten Blick verwundert es daher, daß Bense die Mathematik mit einem Terminus in Verbindung bringt, der Assoziationen an verzerrtes Wissen oder intellektuelle Hirngespinste evoziert. Doch Bense schließt sich hier weder in erster Linie der Marx-Engelsschen noch der von Lukács im Rahmen seiner Totalitarismusstudien entworfenen Ideologiekritik an, welche die Ideologiedebatten der sechziger und siebziger Jahre beeinflußten und die in ihrer Vereinfachung die negative Bedeutung des Begriffs in unserem Alltagsverständnis prägte. Benses Verständnis von Ideologie nähert sich eher jenem von Karl Mannheim an, der in der Fähigkeit, nicht nur die gegnerischen, sondern prinzipiell alle Standorte, auch den eigenen, als ideologisch zu begreifen, die Voraussetzung sah, um von einer bloßen Ideologienlehre zu einer Soziologie des Wissens vorzudringen. ${ }^{30}$ Mannheim betrachtet Wissens- und Normensysteme nicht in Abhängigkeit zu den Motivationen und Interessen bestimmter Gruppen- oder Individualanschauungen, sondern als Funktionen der Seinslage, als Spezialfall allgemeiner Seinsgebundenheit des Denkens. Vergleichbar vollzieht auch Bense eine Erweiterung des Ideologiebegriffs, indem er das von ihm favorisierte mathematische Denken als ideologisch charakterisiert. Die Ideologie der Mathematik ist für ihn 
eine besondere Form geistiger Tätigkeit, die sich von dem Ideal einer homogenen Welt leiten läßt, die rational durchschaubar und kritisch analysierbar ist, um so progressive Funktionen übernehmen zu können.

Die Idee der »mathesis universalis ", zu der sich Bense bekennt, ist für ihn keineswegs eine für das konkrete Dasein belanglose Gedankenspielerei. Das Festhalten an der Einheit unserer technisch-sozialen-kulturellen Zivilisation durch die Konzeption eines einheitlichen mathematischen Geistes stellt für ihn eine Notwendigkeit dar, will man den Anforderungen der von den Naturwissenschaften und der Technik beherrschten Lebenswelt genügen. Wird diese Einheit aufgekündigt und werden die Wechselwirkungen zwischen den verschiedenen Äußerungen des Geistes negiert, dann schließt man aus der Sicht Benses eine Kontrolle der zunehmend denaturierten Realität von vornherein aus. In diesem Sinne muß man den zweiten Band der Konturen einer Geistesgeschichte der Mathematik als ein Plädoyer für die Zusammenarbeit aller Disziplinen, sei es der Kunst, der Literatur, der Wissenschaften und der Philosophie, bei der Bewältigung von neuen Erkenntnissen und ihren Folgen verstehen. Benses Analyse der Dekonstruktion traditioneller Seh- und Lesegewohnheiten durch den Surrealismus als einen Reflex auf die neuen, noch ungewohnten und der Alltagserfahrung widersprechenden Denkweisen der modernen Physik, als eine Anstrengung, neu entdeckte Realitäten intellektuell beherrschbar zu machen, ist nicht rein kunst- und literaturhistorisch motiviert. Die Koinzidenz von Erscheinungen der Kunst und der Wissenschaft ist für ihn der Beweis, daß die Freiheit des Schöpferischen und die Bestrebungen nach Perfektion und Kalkulierbarkeit in der modernen Zivilisation vereinbar, ja untrennbar miteinander verknüpft sind.

Bense verzichtet in seinen späteren Schriften auf den Begriff "Ideologie der Mathematik «. Dennoch blieb er der Idee einer "mathesis universalis" treu. Seine in den Jahren zwischen 1954 und 1982 ausgearbeitete abstrakte, mathematisch formalisierte Ästhetik, ${ }^{31}$ die er bereits im Nachwort des zweiten Bandes der Konturen einer Geistesgeschichte der Mathematik gefordert hatte, ist ebenso Dokument seiner Verbundenheit mit Leibniz wie sein Eintreten für eine Naturphilosophie auf der Basis mathematischer Logik ${ }^{32}$ oder seine Schriften zur Zeichentheorie. Auch 
seine Beschäftigung mit der Kybernetik vollzog sich unter dem Leitgedanken der »mathesis universalis ", hatte doch Leibniz die erste Rechenmaschine erfunden, als er versuchte, seine Idealsprache zu mechanisieren.

Denken und alle Manifestationen des Denkens sind für Bense strukturierte Vorgänge und Zustände, die von angebbaren Gesetzen beherrscht werden. Gedankliche Prozesse zu übersetzen und transparent zu machen, das war es, was Bense bereits bei der Abfassung der Konturen einer Geistesgeschichte der Mathematik reizte. Als der Mathematiker Norbert Wiener 1949 seine berühmte Theorie der Kybernetik in dem Werk Cybernetics - or Control and Communication in the Animal and the Machine der Öffentlichkeit präsentierte, fand Bense darin die Bestätigung, daß Denkprozesse sich nicht nur in mathematischer Sprache beschreiben lassen, sondern daß sie auch in technische Prozesse umgewandelt werden können. Aus seinen fundierten Kenntnissen zur Geschichte der Mathematik heraus begann für ihn die Umwandlung von Gedankenprozessen in mechanische Vorgänge nicht erst mit der Entwicklung von elektronischen Rechenmaschinen und der Nachrichtenübertragungstechnik. Wie er in seinem Artikel »Kybernetik oder die Metatechnik einer Maschine « darlegt, sieht er bereits in Galileis Verlagerung der Naturbetrachtung ins Laboratorium und dem Rückzug auf das Experiment den Beginn der Reproduktion geistiger Akte auf materieller Ebene. Aus dieser Perspektive betrachtet, wurde bereits in der Renaissance der erste Schritt hin zu Überlegungen getan, wie mathematisches Denken technisch nachgebildet werden kann. Meilensteine auf diesem Weg sind Pascals und Leibniz' Rechenmaschinen und der Plan des englischen Mathematikers Charles Babbage zu einer Maschine, die als Speichermedium und für die Programme Lochkarten nutzen sollte und derart bereits die Funktionen moderner Datenverarbeitungssysteme vorweggenommen hatte, die aber erst 50 Jahre später mit den Zähl-, Sortier- und Tabelliermaschinen des amerikanischen Ingenieurs Hermann Hollerith realisiert und bei der Volkszählung 1890 in den USA eingesetzt werden konnten.

Die Ermittlung und Verarbeitung großer Datenmengen, die mit der Lochkartentechnik möglich wurde, hatte nicht nur positive Seiten. Man denke nur daran, daß im zweiten Weltkrieg die 
Lochkartentechnik in Deutschland zu einem wirkungsvollen Überwachungs- und Planungsinstrument avanciert war. Bei aller Faszination, die Bense für die neue Technik und die ihr zugrundeliegende Theorie empfand, war er lange, bevor auf jedem Schreibtisch ein Personal Computer stand und der Informationsund Datenaustausch mehr und mehr auf elektronische Übermittlung umgestellt wurde, lange bevor jemand an Cyberwelten dachte, ein Warner vor den Gefahren des Mißbrauchs dieser Entwicklung. Bereits in seinen frühen technikphilosophischen Arbeiten hatte Bense immer wieder betont, daß die Bedrohung des Menschen durch die Technik nur dann abgewendet werden kann, wenn die rationalen Waffen ausgebildet werden, mit denen die Technik geistig zu beherrschen ist. ${ }^{33}$ Das Eindringen der Technik in Bereiche, die den Unterschied zwischen Materiellem und Nichtmateriellem verwischen, führt in seinen Augen nicht nur zu den gravierendsten Veränderungen in den Lebensverhältnissen des Menschen seit der industriellen Revolution. Die dabei heraufbeschworenen Gefahren scheinen ihm weit verborgener und gefährlicher. Doch genauso wenig wie Norbert Wiener in seiner kritischen Auseinandersetzung mit der Kybernetik unter dem Titel The Human Use of Human Beings (Cybernetics and Society) aus dem Jahre 1949 die positiven Möglichkeiten der neuen Technologie auf Gebieten, die bis dahin als Domäne des Menschen galten, bestreitet, stellt für Bense ein Rückzug der Intellektuellen aus der technischen Welt keine Lösung dar. Die Koexistenz von Technik und Leben sei zwar physisch und metaphysisch zu einem Problem geworden und mit der Existenz von Denkmaschinen glaube man das Vorrecht des Menschen angetastet, schreibt Bense in seinem Vorwort zu der deutschen Ausgabe von Louis Couffignals Les Machines à Penser. Er gibt aber zu bedenken, daß es nur einen Anfangspunkt gebe, der das Problem der Lösung entgegenbringe: man müsse sich entschließen, von der Sache Kenntnis zu nehmen. ${ }^{34}$ Mit seinem Aufsatz »Kybernetik oder Metatechnik einer Maschine" in der Zeitschrift Merkur hatte Bense gezeigt, daß er gewillt war, sich der Herausforderung zu stellen, und daß er bemüht war, die neue alles umwälzende Technologie anderen zur Kenntnis zu bringen.

Bense nennt sich von Hause aus Physiker und Mathematiker. Dies kann nur richtig verstehen, der von der Koinzidenz der 
modernen Physik mit der Mathematik und vom Umschlag der Technik ins Immaterielle weiß. Die Physik hatte eine Höhe der Abstraktion erreicht, bei der die strikte Trennung zwischen realer und möglicher Wirklichkeit aufzuweichen begann. Die Technik hatte einen Grad an Perfektion erlangt, der die Grenzen zwischen Mensch und Maschine neu definierte. Mit der modernen Physik und der Kybernetik erfüllt sich für Bense das Wesen des Geistigen im "Schöpferischen " 35 . Das Abenteuer des Geistes hatte für Bense viele Facetten, doch kann man seine philosophischen, zeichentheoretischen und literarischen Schriften nur dann adäquat einschätzen, wenn man von der Bedeutung der modernen Mathematik und Physik für sein Werk weiß. Heutzutage ist es in der Wissenschaftsgeschichte selbstverständlich geworden, Stile in der Mathematik ${ }^{36}$ oder die gegenseitige Beeinflussung von Computer, Denken und kultureller Entwicklung zu untersuchen, ${ }^{37}$ Max Bense jedoch beschäftigte sich mit dieser Thematik, lange bevor die damit verbundenen Probleme ins allgemeine Bewußtsein gedrungen waren. Besonders seine frühen Schriften zur Mathematik, Physik und Kybernetik können als Pionierleistungen gelten, die heute noch Anstöße zur Beschäftigung mit virulenten Problemen der Zeit bieten. 